\title{
Damping of Power System Oscillation using robust thyristor-controlled phase shifter controller
}

\author{
Ki Sun Han ${ }^{1}$, Moon Gyu Jeong ${ }^{1}$, Jeong Phil Lee ${ }^{2}$ \\ Power Transmission Laboratory, Korea Electric Power Corporation Research Institute, Daejeon, Korea ${ }^{1}$ \\ Subdivision of New \& Renewable Electricity, Kyungnam College of Inform. \& Tech., Busan, Korea ${ }^{2}$
}

\begin{abstract}
In this paper, a robust supplementary controller of thyristor-controlled phase shifter(TCPS) using $H_{\infty}$ control theory has been designed to damp power system oscillation and to enhance power system stability despite uncertainties of the power systems. Since an order of a designed controller using $H_{\infty}$ control theory become very high, a structure-specified low-order TCPS controller using genetic algorithm (GA) has been designed. In order to treat disturbance attenuation and robust stability specifications simultaneously in the stage of $H_{\infty}$ controller design using GA, two weighting transfer functions have been selected. The $H_{\infty}$ norm and damping ratio have been used as the objective function in GA. To verify control performance of proposed TCPS controller, the modal analysis and nonlinear simulations for one-machine infinite bus system have been performed. The simulation results under various disturbances for various operating conditions showthat the proposed TCPS controller provided better dynamic responses in comparison with the conventional PSS and TCPS controller.
\end{abstract}

Keywords: Thyristor-controlled phase shifter (TCPS), $H_{\infty}$ control theory, genetic algorithm (GA), robust control, power system stabilizer (PSS).

\section{INTRODUCTION}

In recent, power systems are very complicatedly interconnected system which includes nonlinear and time-varying elements, and have a small signal oscillatory instability characteristics caused by insufficient natural damping in the system. A power system stabilizer (PSS) has been widely used to solve the oscillatory instability oscillation and to improve small signal stability through the supplementary excitation control of a generator [1-4]. However, a PSS may cause great variations in the voltage profile, may result in leading power factor and may not be able to suppress oscillation resulting from severe disturbance [5]. With the recent advances in power electronics technology, the flexible AC transmission system (FACTS) devices [6 10] and energy storage system (ESS) [11-12] have been installed to enhance power system stability. The application of supplementary controllers for the FACTS devices which use reliable and high speed power electronic devices can considerably improve system damping and can also improve system voltage profile, which is advantageous over PSS [5]. As one of the FACTS devices, the TCPS provides an alternative to the conventional PSS in suppressing power system oscillations and is of particular importance to power system where a PSS cannot be properly installed for reasons such as the system voltage profile, the unsuitability of the generator and so on [13]. The several researches on a supplementary controller for a TCPS have been carried out to enhance power system stability by increasing the damping in power system [13-20]. However, the power systems have system uncertainties due to various generating and loading conditions, variation of system parameters, and system nonlinearity, etc. The increase of these uncertainties makes the optimal control of the power system difficult. The TCPS controller design without considering system uncertainties may fail to operate and stabilize the power system.

In this paper, the robust supplementary controller of TCPS using $H_{\infty}$ control theory [21-22] has been designed in order to improve the robustness of the TCPS controller against system uncertainties. The designed controller using $H_{\infty}$ control theory can achieve robust performance since the unstructured uncertainties of the power system can be included at the controller design stage. However, an order of designed controller becomes very high order and structure of the controller become very complicated. In order to solve the problem, a structure-specified low-order TCPS controller using genetic algorithm (GA) [23] has been designed. The purpose of a structure-specified $H_{\infty}$ controller using GA is to minimize the $H_{\infty}$ norm of the closed loop transfer function including the weighting transfer function and to maximize the damping ratio of the closed loop system including the designed controller. In order to treat disturbance attenuation and robust stability specifications simultaneously in the stage of $H_{\infty}$ controller design using GA, two 
weighting transfer functions have been selected. The $H_{\infty}$ norm and damping ratio have been used as the objective function in GA. The dynamic characteristic responses by means of time domain simulations have been investigated to verify the robustness of the TCPS controller under various operating conditions. The control characteristics of the proposed TCPS controller have been compared with that of the simulated annealing based PSS [8] and the SA based TCPC [8] controller. The simulation results show that the proposed TCPS controller provided better dynamic responses in comparison with the SA-based PSS and SA-based TCPS controller.

\section{POWER SYSTEM MODEL}

2.1 Generator and exciter

Fig. 1 shows the single machine infinite bus power system which has the TCPS and local load admittance Y in a generator bus, and transmission line impedance Z [8][17]. The generator with the IEEE Type-ST1 excitation system is modelled by four first-order nonlinear differential equations as following equations [3].

$$
\begin{aligned}
& \left.\frac{d}{d t} \omega=P_{m}-P_{e}-D(\omega-1)\right) / M \\
& \frac{d}{d t} \delta=\omega_{b}(\omega-1) \\
& \frac{d}{d t} E_{q}^{\prime}=\left(E_{f d}-\left(x_{d}-x_{d}{ }^{\prime}\right) i{ }_{d}-E_{q}{ }^{\prime}\right) / T{ }_{d 0} 0^{\prime}(3) \\
& \frac{d}{d t} E_{f d}=\left(K_{A}\left(V_{r e f}-v_{t}+u_{E}\right)-E_{f d}\right) / T_{A}
\end{aligned}
$$

where $\omega$ is the rotor speed, $\omega_{b}$ is the synchronous speed and $\delta$ the rotor angle. $M$ isinertia constant, $D$ is damping coefficient, $T_{d o}$ ' is the open circuit field time constant and $E_{q}{ }^{\prime}$ is internal voltage. $E_{f d}$ is the field voltage, $v_{t}$ is terminal voltageand $u_{E}$ is PSS controlinput.

The output electric power equation of generator is given by

$$
P_{e}=v_{d} i_{d}+v_{q} i_{q}
$$

where,

$$
\begin{gathered}
v_{t}=\left(v_{d}^{2}+v_{q}^{2}\right)^{1 / 2} \\
v_{d}=x_{q} i_{q}(7) \\
v_{q}=E_{q}^{\prime}-x_{d}^{\prime} i_{d}(8)
\end{gathered}
$$

\subsection{TCPS model}

Fig. 2 shows the configuration of a TCPS [11][15][17]. The basic function of a TCPS is to control transmission line power flow through the modulation of the phase angle difference between the two sides of the transmission line voltage. The voltage at the end bus of TCPS $v^{\prime}$ is phase shifted with respect to the input voltage $v_{t}$ by angle $\Phi$ as shown in Fig. 2 [17].

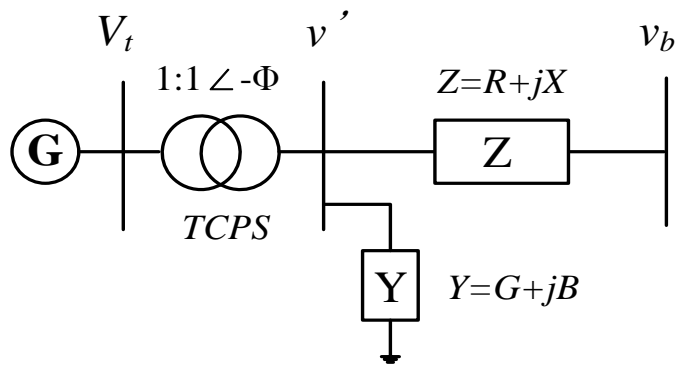

Fig. 1 Single machine infinite bus system with a TCPS

The TCPS in Fig. 1 has a ratio of 1:1 $\angle-\Phi$. The real power flow is obtained by

$$
P_{e}=\frac{E_{q}{ }^{\prime} v_{b}}{X} \sin (\delta-\Phi)(9)
$$


Where $\Phi$ is phase shift angle of the TCPS. The TCPS can control the relative phase angle between the system voltage by adjusting the angle $\Phi$. The phase shift angle of the TCPS, ${ }^{\Phi}$, can be modelled by the first order transfer function and can be written as following equation [8][19].

$$
\frac{d}{d t} \Phi=\left(K_{S}\left(\Phi_{r e f}-u_{T C P S}\right)-\Phi\right) / T_{S}(10)
$$

where $\Phi_{r e f}$ is the reference angle, and $T_{S}$ and $K_{S}$ are the gain and time constant of the TCPS respectively.

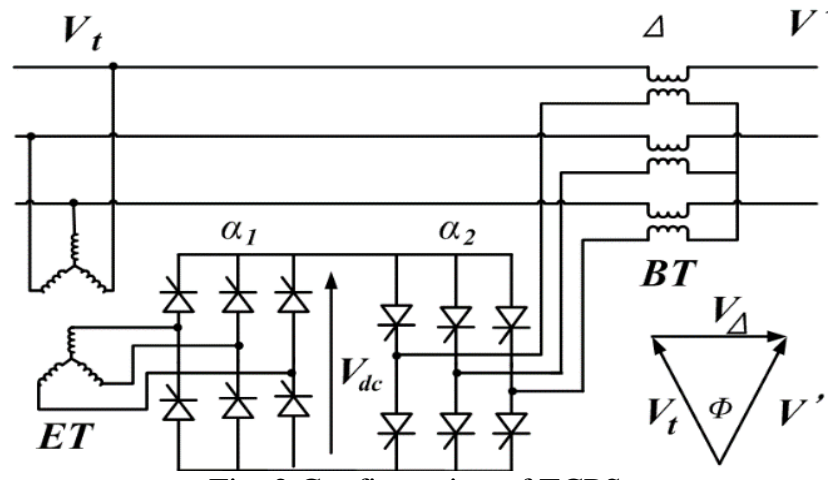

Fig. 2 Configuration of TCPS

\subsection{Linearized model}

To design $H_{\infty}$ controller, the linearized model around a nominal operating point is needed. The detail procedure is in [8]. The linearized equation to nonlinear differential equations such as (1) (4) and (10) including the generator, excitation system and TCPScan be expressed by

$$
\Delta \dot{x}=A \Delta x+B \Delta u(11)
$$

where,

$$
\begin{aligned}
& A=\left[\begin{array}{ccccr}
0 & \omega_{b} & 0 & 0 & 0 \\
-\frac{K_{1}}{M} & -\frac{D}{M} & -\frac{K_{2}}{M} & 0 & -\frac{K_{p}}{M} \\
-\frac{K_{4}}{T_{d o}} & 0 & -\frac{K_{3}}{T_{d o}} & \frac{1}{T_{d o}} & -\frac{K_{q}}{T_{d o}{ }^{\prime}} \\
-\frac{K_{5} K_{A}}{T_{A}} & 0 & -\frac{K_{6} K_{A}}{T_{A}} & -\frac{1}{T_{A}} & -\frac{K_{A} K_{v}}{T_{A}} \\
0 & 0 & 0 & 0 & -\frac{1}{T_{A}}
\end{array}\right] \quad B=\left[\begin{array}{c}
0 \\
0 \\
0
\end{array}\right] \\
& \Delta x=\left[\Delta \delta, \Delta \omega, \Delta E_{q}{ }^{\prime}, \Delta E_{f d}, \Delta \Phi\right]^{T}, \Delta u=\left[u_{T C P S}\right]
\end{aligned}
$$

$\Delta x$ and $\Delta u$ are the state and control vector respectively. A and $\mathrm{B}$ are constant matrices which depend on system parameters and the operating point. The parameters $K_{1} \sim K_{6}, K_{p}, K_{q}$ and $K_{v}$ are computed in operating condition. The block diagram of the linearized power system is shown in Fig. 3 [8][19].

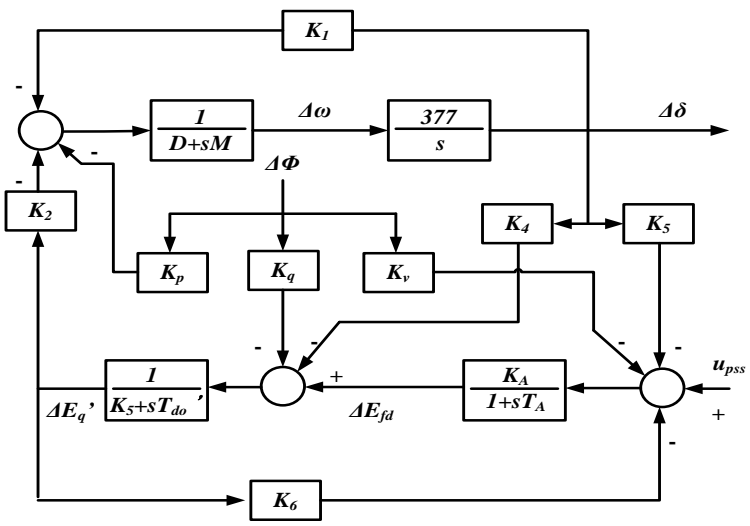

Fig. 3Block diagram of the linearized power system model 


\section{III.DESIGN OF ROBUST TCPS CONTROLLER USING GA}

$3.1 H_{\infty}$ control theory

Fig. 4 shows the block diagram with weighting function $W_{S}(s)$ and $W_{T}(s)$ for $H_{\infty}$ controller design. The augmented transfer function $P(s)$ with weighting function can be expressed by

$$
P(s)=\left[\begin{array}{ll}
P_{11}(s) & P_{12}(s) \\
P_{21}(s) & P_{22}(s)
\end{array}\right]=\left\{\begin{array}{cc}
W_{s}(s) & -W_{s}(s) G(s) \\
0 & W_{T}(s) G(s) \\
I & -G(s)
\end{array}\right](12)
$$

The closed loop transfer function including controller $K_{T C P S}(s)$ from the $\mathbf{w}$ to $\mathbf{z}$ as shown in Fig. 4, linear fraction transformation $F_{l}(P, K)$, can be expressed by

$$
F_{l}(P, K)=\left\lfloor\begin{array}{l}
W_{S}(s) S(s) \\
W_{T}(s) T(s)
\end{array}\right]
$$

where $S(s)$ and $T(s)$ are a sensitivity function and complementary sensitivity function, respectively.

The $H_{\infty}$ control design problem is to find a controller transfer function $K_{T C P S}(s)$ that minimizes the norm of transfer function $F_{l}(P, K)$ from the external disturbance $\mathbf{w}$ to the output $\mathbf{z}$ as shown in Fig. 4, under the condition that the controller $K_{T C P S}(s)$ stabilizes the plant $G(s)$. However, since the structure of the controller is specified as $K_{T C P S}(s)$ in this paper, the $H_{\infty}$ norm of transfer function $F_{l}(P, K)$ must be calculated according to the coefficient of the TCPS controller. The objective in this paper is to find controller $K_{T C P S}(s)$ parameters which minimize the $H_{\infty}$ norm of the transfer function $F_{l}(P, K)$ including the weight function as shown in (14).

$$
\text { minimize }\left\|F_{l}(P, K)\right\|_{\infty}
$$

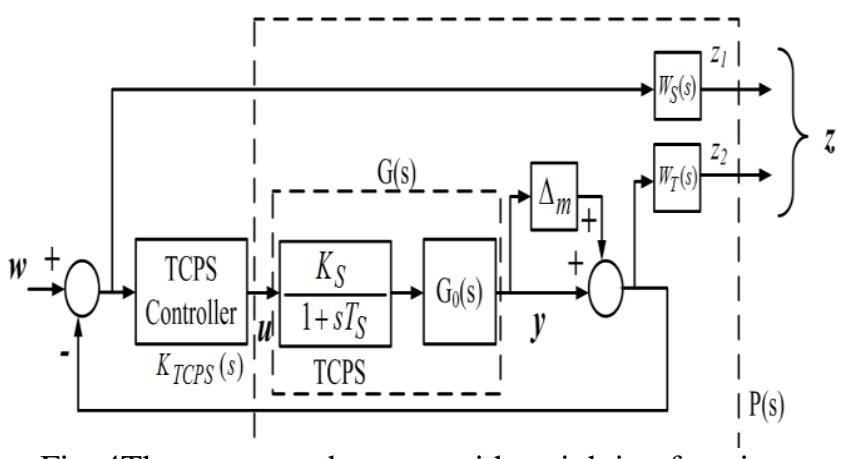

Fig. 4The augmented system with weighting functions

3.2Robust TCPS controller design using GA

The weighting functions in this paper are chosen to be

$$
\begin{aligned}
W_{S}(s) & =\frac{0.34(1.6 s+2.2)}{(s+1)}(15) \\
W_{T}(s) & =\frac{4.17(s+100)}{s+600}
\end{aligned}
$$

The structure of TCPS controller $K_{T C P S}(s)$ has been specified as shown in Fig. 5. To select parameters of $K_{T C P S}(s)$ which minimize $F_{l}(P, K)$ norm as shown in (14), GA has been used. The optimized parameters using GA are $K_{T C P S}$, $T_{w}, T_{T C P S}{ }_{1}, T_{T C P S} 2, T_{T C P S} 3$ and $T_{T C P S}{ }_{4}$ as shown in Fig. 5. In order to optimize these 6 parameters using GA, the objective function has beenchosen as follows. 
Vol. 5, Issue 5, May 2017

$$
\min O J=\alpha 1 / J_{\text {damp }}+\beta\left\|F_{l}(P, K)\right\|_{\infty}
$$

where, $\alpha$ and $\beta$ are the weighting values. $J_{\text {damp }}$ is the damping ratio for the dominant oscillation mode.

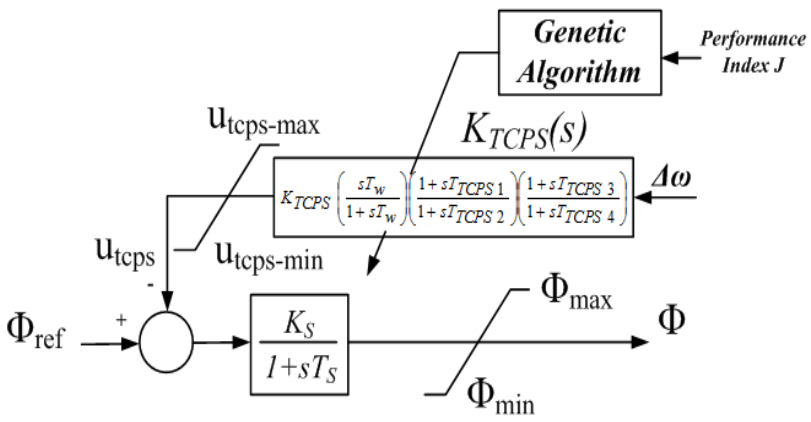

Fig. 5 A Block diagram for selecting the TCPS controller parameters using GA

Assuming that the eigenvalue corresponding to the dominant oscillation mode for the closed loop system is $\lambda=\xi+j \omega$, the damping ratio $J_{\text {damp }}$ is given by,

$$
J_{\text {damp }}=\frac{-\xi}{\sqrt{\xi^{2}+\omega^{2}}}(18)
$$

The goal of this study is to minimize the objective function given by (17). If the eigenvalue of the closed loop system has the positive value, the system is unstable. Then the very large value is assigned to theperformance index (19) by force. The performance index $J$ used to select parameters of TCPS controller using the GA is defined as

$$
\text { Performanc } \quad e \text { Index } J=\frac{1}{\alpha 1 / J_{\text {damp }}+\beta\left\|F_{l}(P, K)\right\|_{\infty}}
$$

\section{IV.SIMULATION RESULTS}

The genetic algorithm optimization toolbox (GAOT) [23] has been used to optimize the parameters of the TCPS controller. To select the parameters of the TCPS controller $K_{T C P S}(s)$ as shown in Fig. 5, the real variable GA usingthe tournament selection method, the arithmetic crossover and the uniform mutation are used. The population size of the GA is 200 and the generation number is 200 . The designed TCPS controller parameters $K_{T C P S}, T_{w}, T_{T C P S} 1, T_{T C P S} 2$, $T_{T C P S} 3$ and $T_{T C P S} 4$ are 180.81, 33.071, 0.070346, 0.017357, 0.11021 and 0.2009 respectively. The calculated $\left\|F_{l}(P, K)\right\|_{\infty}$ with optimal $H_{\infty}$ controller, reference TCPS and proposed TCPS is obtained as 0.748, 0.8605 and 0.748 , respectively. The $\left\|F_{l}(P, K)\right\|_{\infty}$ with proposed TCPS controller using GA has been minimized to the same value as optimal $H_{\infty}$ controller.

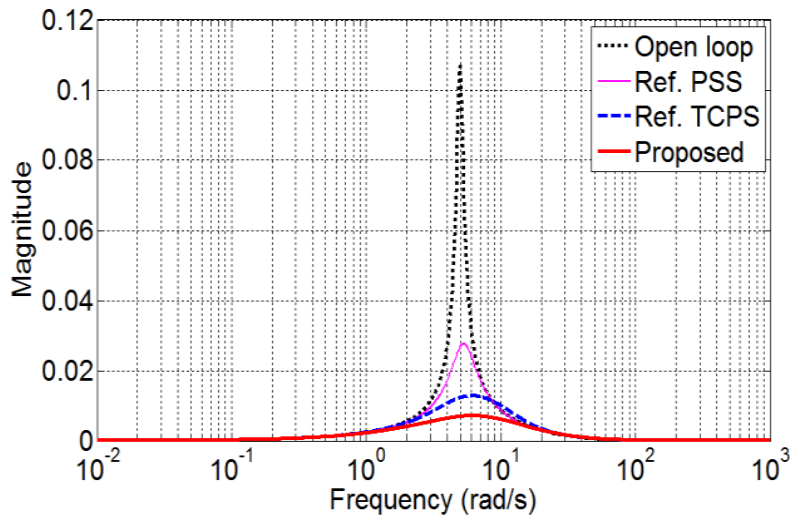

Fig. 6 QFT bounds and loop shaping result using GA 
In order to investigate a disturbance attenuation performance of the designed controller, the singular value bode plot for the open loop and $G_{0}(s) /\left(1+K_{T C P S}(s) G(s)\right)$ with the reference PSS[8], reference TCPS [8] and proposed TCPS controller is shown in Fig. 6. It can be seen that the peak value with the designed TCPS controller is much lower than that with the reference PSS and reference TCPS.

To verify the robust performance of the designed TCPS controller using GA, the nonlinear simulations in time domain have been performed under the 3 operating conditions of the power system. The proposed TCPScontroller have been compared to those of the reference PSS [8] and the reference TCPS [8]. The normal load condition is $P_{e}=1$ (p.u.) and $Q_{e}=0.015$ (p.u.), the leading PF load condition is $P_{e}=0.7$ (p.u.) and $Q_{e}=-0.2$ (p.u.), and the heavy load condition is $P_{e}=1.3$ (p.u.) and $Q_{e}=0.2$ (p.u.). The simulation constraints are $|\Phi| \leq 10^{\circ},\left|u_{p s s}\right| \leq 0.2$ (p.u.) and $\left|E_{f d}\right| \leq 7.3$ (p.u.). The system parameters are shown in Table. $1[8]$.

\section{TABLE I POWER SYSTEM PARAMETERS}

\begin{tabular}{|c|l|}
\hline Generator parameters & $\mathrm{M}=9.26, \mathrm{D}=0, T_{d o}{ }^{\prime}=7.76, x_{d}=0.973, x_{d}{ }^{\prime}=0.19, x_{q}=0.55$ \\
\hline Exciter parameters & $K_{A}=50, T_{A}=0.05$ \\
\hline Line parameters & $\mathrm{R}=-0.034, \mathrm{X}=0.997, \mathrm{G}=0.249, \mathrm{~B}=0.262$ \\
\hline TCPS parameters & $K_{S}=1, T_{S}=0.05$ \\
\hline Initial conditions & $P_{e 0}=1.0, Q_{e 0}=0.015, V_{t 0}=1.05$ \\
\hline
\end{tabular}

\subsection{Normal load condition}

Table 2 shows the eigenvalues and damping ratio of closed loop with the reference PSS, reference TCPS and proposed TCPS respectively. The damping ratio with the proposed TCPS controller is improved in comparison with the reference PSS, reference TCPS controller. Fig. 7 shows the simulation results for rotor angle and terminal voltage with the reference PSS, reference TCPS and proposed TCPS under normal load when a 3-phase short circuit occurs near the infinite bus at $1.0 \mathrm{~s}$ and is cleared at $1.1 \mathrm{~s}$ without the power system configuration change. The first swingof rotor angle using the proposed TCPS controller is smaller than that using the reference PSS and reference TCPS. The voltage profile is greatly improved with the reference TCPS and proposed TCPS. The critical clearing time (CCT) is investigated. The CCT with reference PSS, TCPS and proposed TCPS are $1.143 \mathrm{~s}, 1.173 \mathrm{~s}$ and $1.177 \mathrm{~s}$, respectively.

Fig. 8 shows the simulation results for rotor angle and terminal voltage with the reference PSS, reference TCPS and proposed TCPS when the system inertia has been reduced by $30 \%$ of its nominal value while $25 \%$ pulse increase in the input torque has been applied from $1 \mathrm{~s}$ to $4 \mathrm{~s}$. It can be seen the proposed TCPS controller has robust performance to parameter variation of system.

\subsection{Leading PF load condition}

Fig. 9 shows the simulation results for rotor angle and terminal voltage with the reference PSS, TCPS and proposed TCPS under leading PF load when a 3-phase short circuit occurs near the infinite bus at 1.0s and is cleared at $1.1 \mathrm{~s}$ without the power system configuration change. The first swingof rotor angle using the proposed TCPS controller is smaller than that using the reference PSS and reference TCPS.

\subsection{Heavy load condition}

TABLE II EIGENVALUES AND DAMPING RATIO

\begin{tabular}{|c|l|l|}
\hline & \multicolumn{1}{|c|}{ Eigenvalues } & \multicolumn{1}{c|}{ Damping ratio } \\
\hline & $-0.204,-8.439,-19.155$ & 1 \\
Ref. PSS & $-2.776 \pm 5.042$ & 0.482 \\
& $-3.521 \pm \mathrm{j} 6.406$ & 0.482 \\
\hline & $-18.09,-0.209$ & 1 \\
Ref. & $-3.206 \pm 3.702$ & 0.655 \\
TCPS & $-6.67 \pm \mathrm{j} 7.625$ & 0.658 \\
& $-11.171 \pm 0.917$ & 0.997 \\
\hline & $-4.857 \pm \mathrm{j} 3.375$ & 0.821 \\
Proposed & $-11.025 \pm \mathrm{j} 5.072$ & 0.908 \\
TCPS & $-0.0306,-2.902,-37.046$, & 1 \\
& -41.085 & 1 \\
\hline
\end{tabular}


ISO 3297:2007 Certified

Vol. 5, Issue 5, May 2017

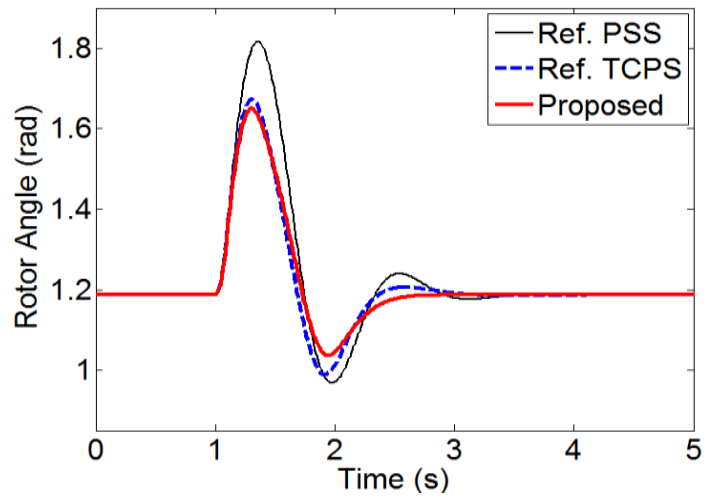

(a) Angular velocity

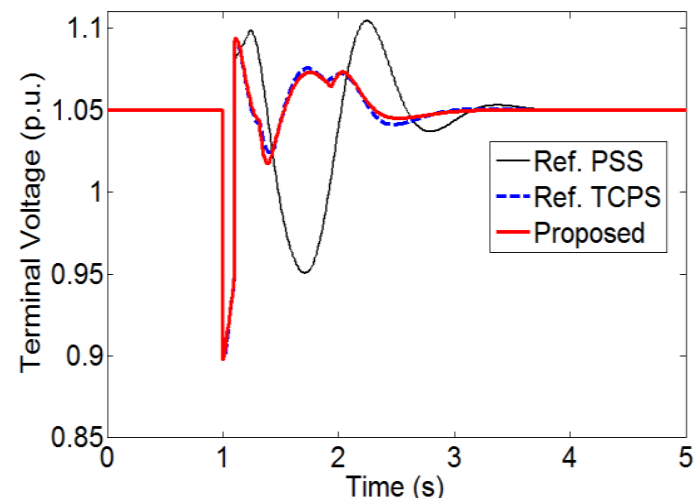

(b) Terminal voltage

Fig. 7. Dynamic responses when a 3-phase short circuit occurs near the infinite bus for 0.1 s under normal load

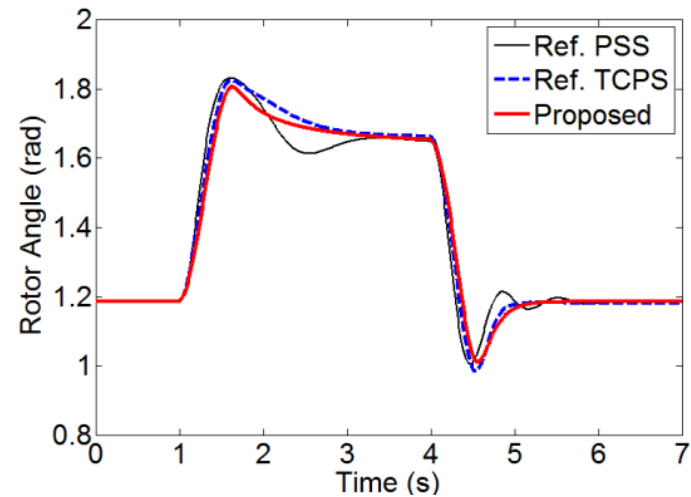

(a) Angular velocity

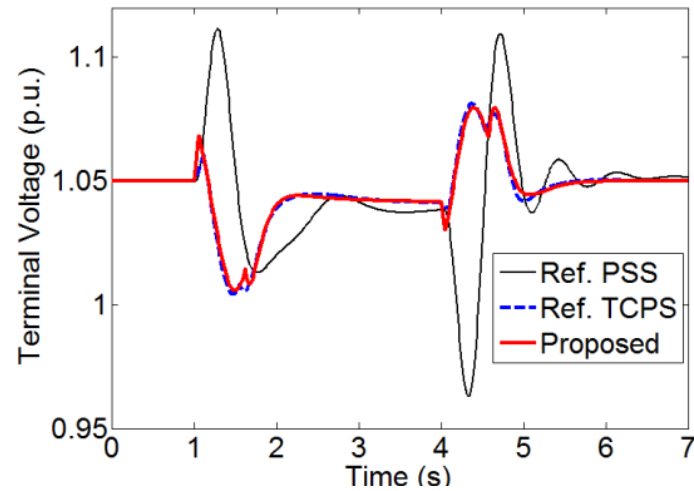

(b) Terminal voltage

Fig. 8. Dynamic responses for the $25 \%$ step increase in input torque for $3 \mathrm{~s}$ under normal load and $30 \%$ reduction of system inertia. 
Fig. 10 shows the simulation results for rotor angle and terminal voltage with the reference PSS, TCPS and proposed TCPS under heavy load when a 3-phase short circuit occurs near the infinite bus at 1.0s and is cleared at $1.05 \mathrm{~s}$ without the power system configuration change. The first swingof rotor angle using the proposed TCPS controller is smaller than that using the reference PSS and TCPS.

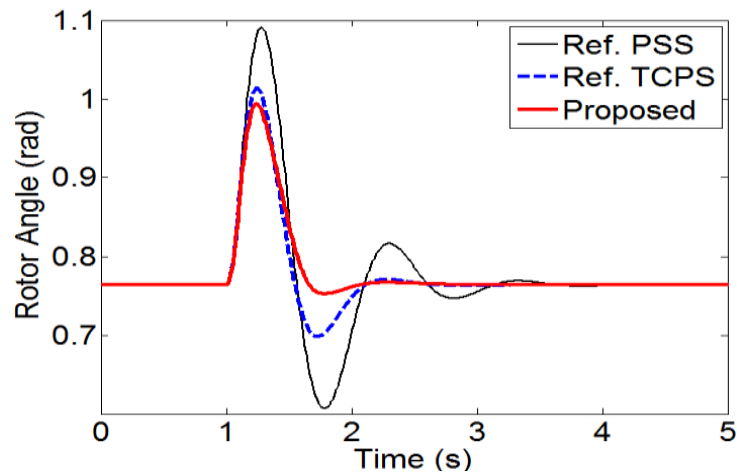

(a) Angular velocity

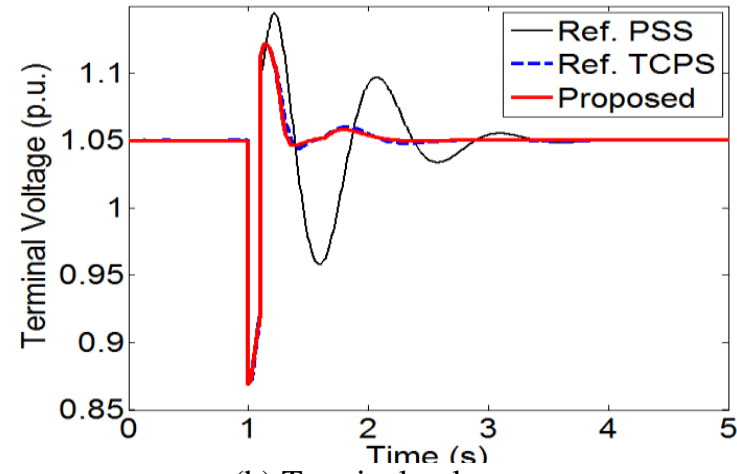

(b) Terminal voltage

Fig. 9. Dynamic responses when a 3-phase short circuit occurs near the infinite bus for 0.1s under leading PF load

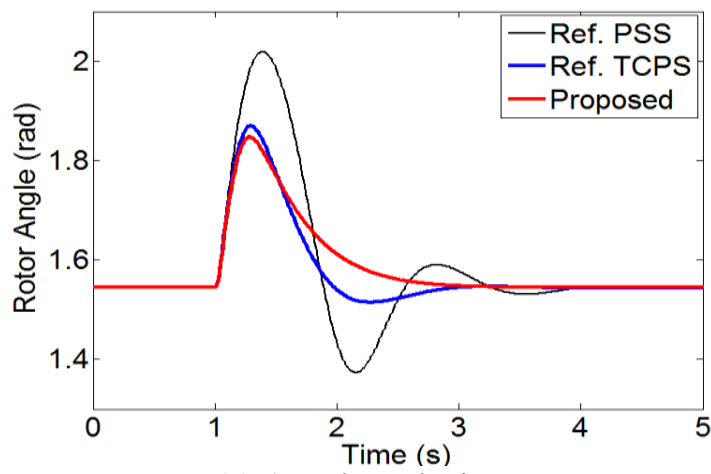

(a) Angular velocity

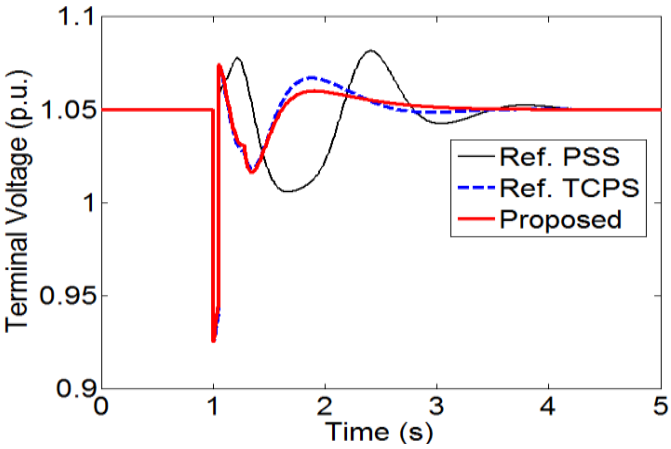

(b) Terminal voltage

Fig. 10. Dynamic responses when a 3-phase short circuit occurs near the infinite bus for 0.05s under heavy load 
Fig. 11 shows the simulations result for rotor angle and terminal voltage with reference PSS, TCPS and proposed TCPS when the initial value of the rotor angle is changed by 0.1 rad under heavy load. It can be seen that the oscillation for rotor angle and voltage profile are damped rapidly comparing with reference PSS, TCPS.

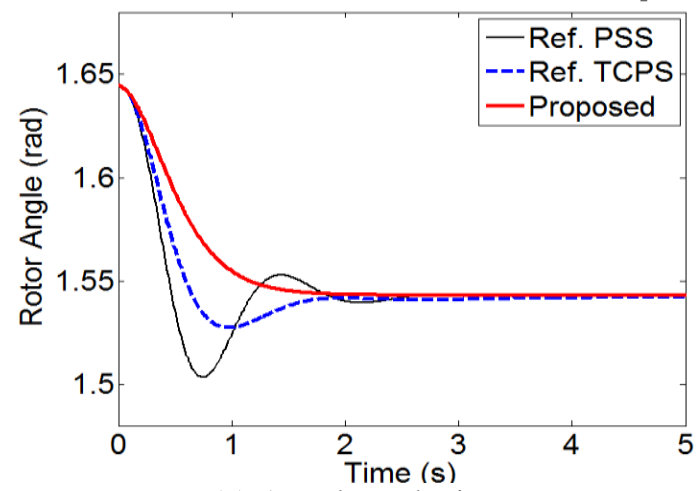

(a) Angular velocity

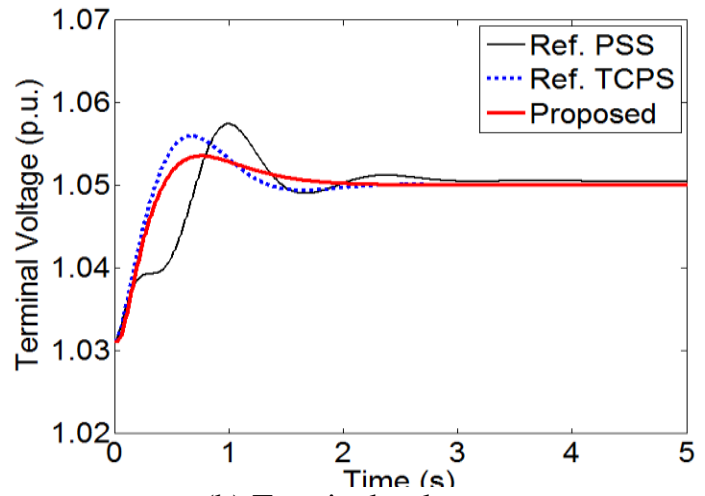

(b) Terminal voltage

Fig. 11. Dynamic responses when a the initial value of the rotor angle is changed by 0.1 rad under heavy load

\section{CONCLUSION}

In this paper, a robust supplementary controller of TCPS using $H_{\infty}$ control theory has been designed to damp power system oscillation and to enhance power system stability despite uncertainties of the power systems. In order to select the parameters of structure-specified robust controller,the $H_{\infty}$ norm and damping ratio as the objective function of GA have been used. The robust performance of the proposed TCPS controller has been compared to those of the conventional PSS and TCPS. The main results in this paper are as follows.

(1The lower order controller through parameter selection of the structure-specified controller using GA can be designed by solving the disadvantages that the order of the $H_{\infty}$ controller becomes high.

(2) The modal analysis results show that the damping ratio with the proposed TCPS is improved in comparison with that of the conventional PSS and TCPS.

3The time domain simulation results show that the proposed TCPS controller is more robust than the conventional PSS and TCPS under various disturbances and parameter variation of power system.

(4)The proposed TCPS controller can more significantly improve the voltage profile comparing with the conventional PSS.

\section{REFERENCES}

[1] F. P. Demello and C. Concordia, “Concept of synchronous machine stability as affected by excitation control,”IEEE Trans. on PAS, vol. 88, no. 4, pp. 316-329, 1969.

[2] P. Kundur, D.C. Lee and H. M. Zein El-Din, "Power system stabilizers for thermal unit: Analytical techniques and on-site validation,"IEEE Trans. on PAS,vol. 100, no. 1, pp. 81-95, 1981.

[3] Yao-nan Yu, Electric power system dynamics, ACADEMIC PRESS, 1983. 


\section{International Journal of Innovative Research in Electrical, Electronics, Instrumentation and Control Engineering}

\section{ISO 3297:2007 Certified}

Vol. 5, Issue 5, May 2017

[4] Y. L. Abdel-Magid and M. A. Abido, "Optimal multi-objective design of robust power system stabilizer using genetic algorithm,"IEEE Trans. on Power System, vol. 18. no.3, pp. 1125-1132, 2003.

[5] A. T. Al-Awami, Y. L. Abdel-Magid and M. A. Abido, "A particle-swarm-based approach of power system stability enhancement with unified power flow controller,"Electrical Power and Energy Systems,vol. 29, pp. 251-259, 2007.

[6] M. A. Abido, "Pole placement technique for PSS and TCSC-based stabilizer design using simulated annealing,"Electrical Power and Energy System, vol. 22, pp. 543-554, 2000.

[7] Q. Zhao and J. Jiang, "Robust SVC controller design for improving power system damping,"IEEE Trans. on Power Systems, vol. 10, no. 4, pp. 1927-1932, 1995

[8] M. A. Abido, "Simulated annealing based approach to PSS and FACTS based stabilizer tuning,"Electrical Power and Energy Systems, vol. 22, pp. 247-258, 2000.

[9] H. F. Wang, "Damping function of unified power flow controller,"IEE Proc.-Gener. Transm. Distrib.,vol. 146, no. 1, pp. 81-87,1999.

[10] H. F. Wang and F. Li F, "Multivariable sampled regulators for the co-ordinated control of STATCOM AC and DC voltage,"IEE Proc.-Gener. Transm. Disturb.,vol. 147, no. 2, pp. 93-98, 2000.

[11] M. H. Wangand H. C. Chen, "Transient stability control of multimachine power systems using flywheel energy injection," IEE Proc.Gener.Transm.Distrib.,vol. 152, no. 5, pp. 589-596, 2005.

[12] W. Du, H. F. Wang, S. Cheng, J. Y. Wen and R. Dunn, "Robustness of damping control implemented by energy storage systems installed in power systems,"Electrical Power and Energy System,vol. 33, pp. 35-42, 2011.

[13] H. F. Wang, F. J. Swift and M. Li, "Analysis of thyristor-controlled phase shifter applied in damping power system oscillations,"Electrical Power and Energy System, vol. 19, no. 1, pp. 1-9, 1997.

[14] Y. L. Tan and Y. Wang, "Nonlinear excitation and phase shifter controller for transient stability enhancement of power systems using adaptive control law,"Electrical Power and Energy Systems, vol. 18, no. 6, pp. 397-403, 1996.

[15] Y. L. Tan and Y. Wang, "Design of series and shunt FACTS controller using adaptive nonlinear coordinated design technique,"IEEE Trans. on Power Systems, vol. 12, no. 3, pp. 1374-1379, 1997.

[16] G. El-Saady, "A variable structure static phase shifting transformer for power system stabilization,” Electric Power Systems Research,vol. 50, pp. 71-78,1999.

[17] M. A. Abido, Thyristor controlled phase shifter based stabilizer design using simulated annealing algorithm, in IEEE Power Tech '99 Conference, Budapest, Hungary, 1999

[18] A. A. Hashmani, Y. Wang and T. T. Lie, "Enhancement of power system transient stability using nonlinear coordinated excitation and TCPS controller,"Electrical Power and Energy Systems, vol. 24, pp. 201-214, 2002.

[19] M. A. Abido, Y. L. Abdel-Magid, “Analysis of power system stability enhancement via excitation and facts-based stabilizers,"Electric Power Components and Systems, vol. 32, pp. 75-91, 2004.

[20] R. J Abraham, D. Das and A. Patra, "Effect of TCPS on oscillation in tie-power and area frequencies in an interconnected hydrothermal power system,"IET Gener. Transm. Distrib. vol. 1, no. 4, pp. 632-739,2007.

[21] K. Glover and J. C. Doyle, "State-space formulae for all stabilizing controllers that satisfy an $H_{\infty}$-norm bound and relations to risk sensitivity," Systems \& Control Letters, vol. 11, no. 167-172, 1988.

[22] J.C. Doyle, K. Glover and P. P. Khargonekar, "State-space solutions to standard $H_{2}$ and $H_{\infty}$ control problems," IEEE Trans. on AC., vol. 34, no. 8, pp. 831-847, 1989

[23] C. Houck, J. Joines, and M. Kay, "A Genetic Algorithm for Function Optimization: A Matlab Implementation,” Technical Report NCSU-IETR-95-09, North Carolina State University, Raleigh, NC. 1995

\section{BIOGRAPHIES}

Ki Sun Han received the M.S. degree in Electrical Engineering from Chungnam National University in 2009. He is currently working for the Korea electric power corporation and its research institute from 1993. His research interests are the energy storage technology, power system stability and substation operation.

Moon GyuJeong received the M.S. and Ph.D. degree in Electrical Engineering from Dong-A University in 2000 and 2005, respectively. He is currently working for the Korea electric power corporation and its research institute from 2001. His research interests are the energy storage technology, power system stability and substation operation.

Jeong Phil Lee received the M.S. and Ph.D. degree in Electrical Engineering from Dong-A University in 1999 and 2002, respectively. He worked from 2005 to 2012 in the Korea electric power corporation research institute. He is currently a professor in Kyungnam College of Information and Technology. His research interests are the energy storage technology, power system stability and new and renewable energy. 